\title{
A prospective study to evaluate the safety and efficacy of symbiotic supplementation (probiotic and prebiotic combination) in stage 5D chronic kidney disease patients
}

\author{
Sanjay Srinivasa ${ }^{1}$, Santhosh K. Madhusudhan ${ }^{2 *}$
}

${ }^{1}$ Department of Nephrology, Sapthagiri Institute of Medical Sciences, Bangalore, India ${ }^{2}$ Department of Pharmacology, Jagadguru Jayadeva Murugarajendra Medical College, Davangere, India

Received: 01 March 2017 Accepted: 14 March 2017

\section{*Correspondence to:}

Dr. Santhosh K. Madhusudhan, Email:

sankumar20123@gmail.com

Copyright: (C) the author(s), publisher and licensee Medip Academy. This is an openaccess article distributed under the terms of the Creative Commons Attribution NonCommercial License, which permits unrestricted noncommercial use, distribution, and reproduction in any medium, provided the original work is properly cited.

\begin{abstract}
Background: Chronic Kidney Disease (CKD) is one of the major health disorders associated with significant morbidity and mortality. This was a 6 week's interventional study of orally administered, symbiotic supplement (probiotic with prebiotic) in stage 5D patients of chronic kidney disease (CKD) on twice a week hemodialysis. The objective was to look for safety of symbiotics (Nitrophage Forte ${ }^{\mathrm{TM}}$ ) and for its anti-inflammatory effects measured by serum hsCRP (Highly specific C reactive protein), IL-6 (Interleukin- 6) and TNF- $\alpha$ (Tumour Necrosis Factor- $\alpha$ ) levels. This translating to improvement in the Quality of life (QOL) assessed using SF-36 QOL questionnaire and Subjective Global Assessment (SGA) scoring.

Methods: Subjects on twice a week dialysis for at least 3 months were included. Parameters at baseline (representing previous 3 months) were compared to that at the end of treatment. Oral supplementation of strain specific and unique composition of symbiotic sachet supplementation were administered twice daily containing Lactobacillus acidophilus 400mg, Bifidobacterium longumm 400mg, Streptococcus thermophilus with Fructooligosaccharide $100 \mathrm{mg}$ adding to 1 gram was given for 6 weeks.

Results: 38 patients out of total 48 enrolled completed the study. Symbiotic therapy was found to be well tolerated with no significant adverse effects. $60.52 \%, 55.26 \%, 44.7 \%$ of the patients had a decrease in hsCRP, TNF- $\alpha$ and IL-6 respectively. Among responders hsCRP and TNF- $\alpha$ showed significant decrease in levels from the baseline ( $p$ <0.05). Modified SF-36 QOL questionnaire mean score revealed significant improvement in general health $(\mathrm{p}$ $<0.05$ ). Among secondary parameters renal biomarkers like urea, BUN and sodium showed statistically significant decrease ( $\mathrm{p}<0.05)$.

Conclusions: This study establishes the safety and anti-inflammatory efficacy of this symbiotic supplement. To our knowledge this is the first study looking at anti-inflammatory role of symbiotic in CKD 5D Patients. A placebo controlled, double blinded study with a larger sample size is warranted in future to further establish these findings.
\end{abstract}

Keywords: Chronic kidney disease, Probiotic, Quality of life, Symbiotic, Uremia

\section{INTRODUCTION}

Chronic Kidney Disease (CKD) is one of the major health disorders and is associated with significant morbidity and mortality. Worldwide more than 20 billion dollars is spent annually for management of CKD patients. Once in stage 5, CKD patients have 1000 fold increased risk of cardiovascular morbidity, mortality and premature death. One of the significant factors contributing to poor outcome among these patients is the high inflammatory status. CKD is a pro-inflammatory state; use of bio-incompatible extracorporeal circuit for hemodialysis worsens this state.

High inflammatory status also predisposes to protein energy wasting and impairs the Quality of life (QOL) 
with decrease in productivity of the patient. Contributing factors of CKD like diabetes, hypertension, obesity, has spiraled out of control increasing the incidence of CKD, which now is being recognized as a major public health concern. ${ }^{1}$ Globally CKD is the 12 th major cause of death and 17th major cause of disability. This would be an underestimate as patients with CKD are more likely to die of CVD than to reach End Stage Renal Disease (ESRD). ${ }^{2}$ In India Modi and Jha have suggested a crude incidence of ESRD at 151 per million and age-adjusted incidence of 232 per million. ${ }^{3}$ In a published "screening and early evaluation of kidney disease study" the prevalence of CKD stage 5 was reported at $0.8 \% .^{4}$

CKD has been a devastating social and economic burden for patients and their families in developing countries like India. A very conservative estimate of the ESRD burden, based on a population of 1.1 billion, is that 16.5 to 22 hundred thousand people develop ESRD in India annually, of whom only $10 \%$ or less receives treatment and the rest die. As per the Indian CKD registry database, every year 3,500 patients undergo Renal Transplants (RT), 3,000 are put on Continuous Ambulatory Peritoneal Dialysis (CAPD) and approximately 15,000 Patients are initiated on maintenance hemodialysis. The renal transplantation rate amounts to paltry 3.25 per million per year. ${ }^{5}$ A person's risk for CKD is determined by both genetic and environmental factors. The significant rise in the prevalence of CKD within a single generation suggests a dominant role of environment in promoting CKD. One of the largest environmental factors a person is exposed to is the food he eats.

The stage 5 CKD patients have high uremia characterized by accumulation of organic waste products, the uremic retention solutes (URSs). ${ }^{6}$ Retention of these nitrogenous toxins affects multiple organ systems causing significant morbidity and mortality. The newer insights into role of gut in the contribution to the uremia and its complications in CKD patients has been only recently uncovered. Recent data highlight that uremia is associated with abnormalities in the gastrointestinal mucosa causing a disequilibrium state in the intestinal biotic ecosystem (Dysbiosis). There is increasing clinical evidence that patients with CKD have a distinctly dysbiotic intestinal gut microbiota.

Dysbiosis in uremia may be attributed to factors likehydrolysis of urea in the gut releasing ammonia, active and excessive secretion of uric acid and oxalate into the colon causing changes in the gut ph. Dietary restrictions in CKD patients of potassium rich products like fruits and vegetables, which are major source of indigestible complex carbohydrates (fibers), the main nutrient for colonic bacteria, affects the growth of normal commensal bacteria leading to proliferation of non- commensal bacteria. $^{7}$

In addition, CKD patients with frequent use of antibiotics, phosphates and potassium binders, oral iron supplements associated with slow colonic transit, metabolic acidosis and intestinal wall edema affect the growth and multiplication of colonic microbiota. ${ }^{7}$ These changes in the gut milieu leads to distinctly dysbiotic intestinal bacterial community contributing to the complications of CKD.

This dysbiosis is characterized by greatly increased counts of both aerobic and anaerobic bacteria, with substantial decrease in both Lactobacillaceae and Prevotellaceae families. ${ }^{8}$ Lower intestinal microbial flora also shows significantly altered composition with overgrowth of aerobic bacteria and significantly lower numbers of Bifidobacteria and higher Clostridium perfringes, confirmed by fecal microbiota analysis of hemodialysis patient. ${ }^{9}$

Dysbiosis then drives a cascade of metabolic abnormalities like:

\section{Inflammation contributed by}

- Loss of integrity or impairment of intestinal barrier by disruption of the colonic epithelial tight junction allowing translocation of mainly gram negative bacteria and lipo-polysaccharide into the system.

- Pathobionts stimulate dendritic cells that activate a Th17/Th1 T-cell response and enhance production of inflammatory cytokines like INF- $\gamma$, IL-1,6 \& TNF- $\alpha$,

It also contributes to profound immunosuppressive state, excess uremic toxin production by altered carbohydrates, protein, and bile acid fermentation leading to progressive kidney failure, protein energy wasting with impaired quality of life and increased cardiovascular morbidity and mortality. ${ }^{10}$

Until recently, the gastrointestinal tract (GIT) and its associated microbiota was a neglected factor in chronic disease development. ${ }^{11}$ It is increasingly recognized that bacterial metabolism and its ensuing metabolites, such as phenols, indoles, and amines, may significantly contribute to uremic toxicity. ${ }^{12}$

It has also been documented that the GIT microbiota may be the key factor that maintains the GIT in a chronic inflammatory state, as in CKD. ${ }^{13}$ Schepers and colleagues have recently proposed that the gut is the forgotten organ in kidney disease and uremia. They argue that, apart from the role of the GIT as a route by which uremic toxins or their precursors enter the body, the small and large bowel also act as active participants through the maintenance of a dysbiotic state. Once a dysbiotic and inflamed GIT ensues then the systemic spread of these molecules becomes possible, augmenting the likelihood of uremic toxin overload. ${ }^{14}$ Hence, a link between the GIT inflammation, dysbiosis, and circulating uremic toxins is established and this is suggestive of a GIT dysbiosisrenal relationship, particularly for the development and 
progression of CKD with associated cardiovascular disease.

In the past 30 years there has been no significant breakthrough in the management of CKD. A number of treatments options have been proposed, unfortunately most of these treatments display inherent disadvantages like side effects, high cost, non-availability for patients with moderate CKD and many restricted still to experimental studies. ${ }^{6}$

Published studies prove probiotics and prebiotics combinations have a therapeutic role in maintaining a metabolically balanced GIT, and reducing progression of CKD and associated uremia. In a hypothesis review by L. Vitetta and G. Gobe et al, they suggested - multi-strain probiotic formulation, with or without prebiotic, is a biologically plausible pharmacobiotic therapy for CKD. Restructuring the GIT microbial community by rescuing from a pro-inflammatory or dysbiotic state may prove beneficial in reducing the uremic load produced in the gut and its escape to the systemic circulation. ${ }^{15}$

Probiotic a word derived from Greek meaning 'for life' is defined by the Food and Agriculture Organization (FAO) and World Health Organization (WHO) as "live microorganisms which when administered in adequate amounts confer a health benefit on the host" (2002). ${ }^{16}$ In contrast, Prebiotic have been defined as those nutritional supplements (like inulin) that favors the growth and increasing survival of probiotic bacteria. ${ }^{17} \mathrm{~A}$ symbiotic preparation is one that combines multi strain probiotic micro-organism with prebiotic compounds.

In the past 5 years there have been significant scientific developments linking gut health and chronic kidney disease. As the gut microbiota is intimately influenced by diet, the discovery of the kidney-gut axis has created new therapeutic opportunities for nutritional intervention involving dietary protein, fibre, prebiotic, probiotic, and symbiotics. ${ }^{10}$ It has also been recently reported that the pharmacobiotic potential of the GIT microbiota may provide a plausible therapeutic avenue with the administration of live multi-strain probiotic cultures.

Following recent explosion of knowledge on the metabolic potential of gut microbiome and its critical role in the pathogenesis of inflammatory state, probiotics now are the new focus of investigations as a possible natural bio-treatment in the management of CKD and its related complications. $^{7}$

More clinical research is needed to evaluate compounds that will re-establish gut microbiota and prevent dysbiosis related complications in CKD. Hence this study aims to evaluate the safety of one such symbiotic and its antiinflammatory effects in treatment of CKD stage 5D patients on twice a week hemodialysis.

\section{METHODS}

\section{Study design}

The study was a prospective, open labeled, interventional, 6 weeks study of orally administered, symbiotic supplement (probiotic with prebiotic) in Stage 5D patients of chronic kidney disease on twice a week hemodialysis for past three months. The study was conducted in department of Nephrology. The study protocol was approved by the institutional ethics committee before commencement of the study. The study was registered under clinicaltrials.gov (CTRI/2015/09/006181).

The objective of the study was to look for safety and efficacy of symbiotic (Nitrophage Forte ${ }^{\mathrm{TM}}$ ), for its antiinflammatory effects measured by serum hsCRP (Highly specific $C$ reactive protein), IL-6 (Interleukin 6), TNF- $\alpha$ (Tumor Necrosis Factor). Role of Symbiotic therapy in improving the Quality of life (QOL) of patients was assessed using SF-36 Quality of Life Questionnaire modified for Renal and Dialysis patients and Subjective Global Assessment (SGA) scoring among patients with stage $5 \mathrm{D}$ CKD on twice a week hemodialysis (HD). ${ }^{18}$ The secondary objective was to look for improvement in other biochemical and hematological parameters of these patients.

\section{Study procedure}

48 patients were enrolled in the study. Patients were recruited from the outpatient Chronic Kidney Disease clinic.

\section{Inclusion criteria}

1. Age above 18 years

2. Ability to give written informed consent

3. Patients with Stage 5D CKD on twice a week hemodialysis (HD) for at least 3 months prior to the enrolment

4. Patients willing to come for regular follow-up visits.

\section{Exclusion criteria}

1. Active infectious conditions in last 30 days

2. Pregnancy or nursing mothers

3. Patients with auto immune diseases or connective tissue disorders

4. Diabetic foot infection

5. History of cardiovascular events

6. Patients already on test drug

7. Anti $\mathrm{HCV}, \mathrm{HbsAg}$ - positivity.

After patients fulfilled the above inclusion and exclusion criteria, the study details were completely briefed by the investigator to the patient and a signed written informed consent was obtained. 
At the baseline visit, complete clinical and physical examination was done and findings were recorded in the case report form. Blood was collected to carry out investigations that included lipid profile (HDL, LDL, triglycerides, total cholesterol), hematological parameters $[\mathrm{Hb} \%, \mathrm{PCV}$, ferritin, total proteins, albumin, blood urea and creatinine, electrolytes (sodium, potassium) and inflammatory markers (hsCRP, IL-6 and TNF- $\alpha$ )].The samples for biochemical analysis at baseline and at completion were collected before the start of dialysis.

The patients were administered Kidney Disease Quality of life (KDQOL-SF36TM) questionnaire and SGA scoring was done by a trained physician/nurse before enrollment and after completion of the study. The scoring at baseline and at end of study was done by the same observer for an individual patient. Throughout the study subjects were maintained on same dialysis protocol, with no changes in diet, medications, activity or dialysis dose.

All patients were asked to take the study product symbiotic (Nitrophage Forte ${ }^{\mathrm{TM}}$ ) sachet supplied by Mylin Biotech India Private Limited, half an hour before food, mixed in $20 \mathrm{ml}$ water at room temperature twice daily for a period of 6 weeks. Symbiotic sachets contained Lactobacillus acidophilus 400mg, Bifidobacterium longumm 400mg and Streptococcus thermophilus 100mg with Fructo-oligosaccride $100 \mathrm{mg}$ adding to $1 \mathrm{gm}$ per sachet. The total treatment period was 6 weeks.

Patients were advised to store the symbiotic in the refrigerator at $<25^{\circ} \mathrm{C}$ degree and not to freeze as recommended by the manufacturer. The drug was given 30 minutes before the meal in $20 \mathrm{ml}$ of normal water, twice daily. Compliance was ensured by weekly twice phone calls to patients and empty sachets were collected on weekly basis. Sample was given to patient to be carried in thermo box to their residence. At the end of the treatment period of 6 weeks, patients visited the hospital for final examination. They were questioned for any adverse reactions and all new symptoms were duly recorded.

Patients were evaluated for treatment compliance followed by complete physical and clinical examination. Blood was drawn to carryout biochemical and hematological investigation. Patients were administered KDQOL-SF ${ }^{\mathrm{TM}}$ questionnaire and SGA scoring by the same observer who administered at time of recruitment. All the collected data was documented .The patients were advised to visit the clinic any time during the study period if he or she had any issues or for any unexpected or new symptom. All blood samples at baseline and at end of study was collected, transported by air and processed by an independent third party, SRL Ranbaxy at their Mumbai NABL, CAP and ISO accredited laboratory for analysis and reporting. IL- 6 and TNF- $\alpha$ was estimated by ELISA. hs CRP was estimated by nephelometry. Total proteins, BUN, triglycerides, total cholesterol, HDL, LDL and creatinine were estimated by spectrophotometer. Ferritin level was estimated by chemiluminescence. The measurable parameters were collected at baseline which represented the steady state of previous 3 months and these parameters were compared to end of study period parameters after 6 wks of interventional therapy (symbiotic supplement). To make the data comparable, no changes were made in the medications, diet, dialysis prescription with its duration or frequency. Physical activity of the subjects was maintained same during the study period. Data from the two time period was compared for (1) safety profile, (2) inflammatory status - measured by markers like hsCRP, IL-6, TNF $\alpha$ and (3) quality of life assessment by SF-36 (modified for renal and dialysis patients) and SGA scoring.

\section{Statistical analysis}

All analysis was carried out by using SPSS software version 18. The biochemical and hematological data are represented as Mean \pm standard deviation. Percentage of floor, percentage of ceiling, and internal consistency reliability (Cronbach's alpha coefficient) were calculated for KDQOL-SF ${ }^{\mathrm{TM}}$ questionnaire. Student's t-test was used to evaluate statistical significance between baseline and end of visit data. A p value < 0.05 was considered statistically significant.

\section{RESULTS}

Table 1: Percentage of patients with change in biochemical and hematological parameter at end of treatment compared to baseline.

\begin{tabular}{|llll|}
\hline \multicolumn{4}{l}{ Inflammatory markers } \\
& $\%$ patients & $\mathrm{N}=38$ & Remark \\
\hline CRP & 60.52 & 23 & Decrease \\
\hline IL-6 & 44.7 & 17 & Decrease \\
\hline Tnf- $\alpha$ & 55.26 & 21 & Decrease \\
\hline Kidney functions & & & \\
\hline Creatinine & 28.9 & 11 & Decrease \\
\hline BUN & 34.21 & 13 & Decrease \\
\hline Urea & 28.94 & 11 & Decrease \\
\hline MDRDGFR & 52.63 & 20 & Increase \\
\hline CGGFR & 60.52 & 23 & Increase \\
\hline Albumin & 65.78 & 25 & Increase \\
\hline Total-proteins & 57.89 & 22 & Increase \\
\hline Blood parameters & & & \\
\hline Hb & 55.26 & 21 & Increase \\
\hline Hematocrit & 57.89 & 22 & Increase \\
\hline Ferritin & 56.66 & 17 & Increase \\
\hline Na ${ }^{+}$ & 47.36 & 18 & Decrease \\
\hline $\mathrm{K}^{+}$ & 47.36 & 18 & Decrease \\
\hline Lipid profile & & & \\
\hline Triglycerides & 39.47 & 15 & Decrease \\
\hline Total cholesterol & 52.63 & 20 & Decrease \\
\hline LDL & 42.1 & 16 & Decrease \\
\hline HDL & 50 & 19 & Increase \\
\hline & & & \\
\hline
\end{tabular}


Among the 48 patients enrolled in the study, 38 patients completed the study as per protocol. Of the 10 patients excluded from the efficacy analysis, 4 were lost to followup and 6 patients had not taken study medication as per protocol. However all patients enrolled in to the study were included in the safety analysis. The mean age of the patients who completed the study was 50.23 years, $65 \%$ were males. The percentage of patients with change in biochemical and hematological parameters and the percentage change in these parameters at end of visit compared to baseline with $\mathrm{p}$ values are mentioned in Table 1 and 2 respectively.

Table 2: Percentage of change in biochemical and hematological parameters at end of treatment compared to baseline in all patients and responders.

\begin{tabular}{|c|c|c|c|c|c|}
\hline & All patients & p value & Responder & p value & Remark \\
\hline CRP & 6.13 & 0.13 & 18.55 & 0.02 & Decrease \\
\hline IL-6 & 29.72 & 0.36 & 69.7 & 0.05 & Decrease \\
\hline TNF- $\alpha$ & 9.22 & 0.2 & 33.41 & 0.005 & Decrease \\
\hline \multicolumn{6}{|c|}{$\%$ change in kidney functions } \\
\hline Creatinine & 6.12 & 0.13 & 18.55 & 0.33 & Decrease \\
\hline BUN & 16.71 & 0.002 & 12.81 & 0.02 & Decrease \\
\hline Urea & 20.28 & 0.002 & 15.71 & 0.03 & Decrease \\
\hline MDRDGFR & 5.13 & 0.52 & 16.21 & 0.009 & Increase \\
\hline CGGFR & 2.3 & 0.29 & 12.05 & 0.01 & Increase \\
\hline Albumin & 0.93 & 0.72 & 8.41 & 6.98 & Increase \\
\hline Total Proteins & 1.14 & 0.25 & 5.42 & 4.89 & Increase \\
\hline \multicolumn{6}{|c|}{$\%$ change in Blood parameters } \\
\hline $\mathrm{Hb}$ & 0.43 & 0.27 & 8.86 & 0.002 & Increase \\
\hline Hematocrit & 0.75 & 0.81 & 10.49 & 0.0001 & Increase \\
\hline Ferritin & 19.08 & 0.33 & 91.32 & 0.02 & Increase \\
\hline $\mathrm{Na}^{+}$ & 1.22 & 0.02 & 1.51 & 0.0002 & Decrease \\
\hline $\mathrm{k}^{+}$ & 1.8 & 0.67 & 18.49 & 0.0001 & Decrease \\
\hline \multicolumn{6}{|c|}{$\%$ Change in lipid profile } \\
\hline Triglycerides & 7.16 & 0.43 & 31.94 & 0.0001 & Decrease \\
\hline Total Cholesterol & 0.16 & 0.96 & 12.68 & 0.0001 & Decrease \\
\hline LDL & 0.9 & 0.85 & 17.36 & 0.015 & Decrease \\
\hline HDL & 2.06 & 0.57 & 17.81 & 0.0001 & Increase \\
\hline
\end{tabular}

\section{Inflammatory markers}

hsCRP- $60.52 \%$ of the patients had a decrease in hsCRP. Overall in all patients there was decrease in hsCRP level by $6.13 \%$.When only responders were analyzed there was a significant decrease in hsCRP values by $18.55 \%$ $(\mathrm{p}<0.02)$.

TNF $\alpha-55.26 \%$ of study patients had decrease in TNF- $\alpha$. There was $9.22 \%$ decrease in TNF levels from baseline by the treatment. The responders showed a significant decline in levels by $33.41 \%$ from baseline ( $\mathrm{p}<0.005)$. IL$6-44.7 \%$ of study patients had decrease in IL-6. There was $29.72 \%$ decrease in IL-6 levels from baseline by the treatment. The responders showed a significant decline in levels by $69.7 \%$ from baseline, $\mathrm{p}<0.05$.

\section{Hematological and Biochemical Parameters}

$34.21 \%$ and $28.94 \%$ patients showed significant decrease in BUN and urea respectively, $\mathrm{p}<0.05$. The responders showed a significant decrease in BUN and Urea levels by $12.8 \%$ and $15.7 \%$ respectively from baseline, $\mathrm{p}<0.05$. $55.26 \%$ and $57.89 \%$ of patients had increase in $\mathrm{Hb} \%$ and hematocrit respectively, though not significant. However the responders showed a significant increase in $\mathrm{Hb} \%$ and hematocrit levels by $8.86 \%$ and $10.49 \%$ respectively from baseline, $\mathrm{p}<0.05$.

$52.63 \%$ and $60.52 \%$ patients showed increase in MDRD GFR and cGFR respectively. The responders showed a significant increase in MDRD GFR and cGFR by 16.21 and $12.05 \%$ respectively, $\mathrm{p}<0.05$. The clinical significance of the same needs to be evaluated in further randomized studies with stage 3 and $4 \mathrm{CKD}$. More than $50 \%$ of the patients had an increase in total protein and albumin levels, though not statistically significant .The results are encouraging and may have been flawed by short duration of study period. Assumption may be that, it might gain significance, if the treatment could have been extended beyond 3 months. $39.47 \%, 52.63 \%$ and $42.1 \%$ of the study subjects showed decrease in triglycerides, 
total cholesterol and LDL levels respectively. Responders among the group showed significant decrease in levels by $31.94 \%, 12.68 \%, 17.36 \%$ respectively, $\mathrm{p}<0.05$. Similarly $50 \%$ of patients showed improvement in HDL levels, though not significant. Responders among the group showed significant increase by $17.81 \%$ from the baseline.
Renal biomarkers like urea, BUN and sodium showed statistically significant decrease, $\mathrm{p}<0.05$. $47.36 \%$ of patients had a decrease in potassium levels though not significant, among responders there was a significant decrease by $18.49 \%$ from baseline levels, $\mathrm{p}<0.0001$.

Table 3: Central tendency, variability (including floor and ceiling effects), reliability of KDQOL-SFTM scales at baseline visit.

\begin{tabular}{|lllllll|}
\hline Kidney disease-targeted scales & $\begin{array}{l}\text { No of } \\
\text { items }\end{array}$ & Mean & $\begin{array}{l}\text { Std. } \\
\text { deviation }\end{array}$ & $\begin{array}{l}\% \\
\text { floor }\end{array}$ & $\%$ Ceiling & $\begin{array}{l}\text { Internal consistency } \\
\text { reliability }\end{array}$ \\
\hline Burden of kidney disease & 5 & 31.87 & 21.01 & 2.63 & 0.00 & 0.449 \\
\hline Cognitive function & 3 & 62.21 & 21.95 & 2.63 & 0.00 & 0.471 \\
\hline Effect of kidney disease & 6 & 69.47 & 21.87 & 2.63 & 10.53 & 0.504 \\
\hline Sleep & 4 & 66.47 & 20.86 & 0.00 & 2.63 & 0.484 \\
\hline Quality of social interaction & 3 & 59.58 & 23.00 & 0.00 & 10.53 & 0.451 \\
\hline Symptom/problem list & 11 & 59.37 & 19.75 & 0.00 & 2.63 & 0.488 \\
\hline Social support & 2 & 65.76 & 31.72 & 5.26 & 31.57 & 0.392 \\
\hline Work status & 2 & 32.89 & 33.44 & 44.74 & 13.16 & 0.557 \\
\hline 36-item health survey & & & & & & \\
\hline Physical functioning & 10 & 45.21 & 27.11 & 7.90 & 5.30 & 0.871 \\
\hline Role-physical & 4 & 25.87 & 35.13 & 52.60 & 13.20 & 0.844 \\
\hline Pain & 2 & 48.79 & 29.56 & 5.30 & 10.50 & 0.842 \\
\hline General health & 5 & 34.63 & 20.93 & 2.60 & 0.00 & 0.838 \\
\hline Emotional well-being & 5 & 57.61 & 20.94 & 2.60 & 2.60 & 0.851 \\
\hline Role-emotional & 3 & 37.71 & 45.97 & 55.30 & 2.60 & 0.839 \\
\hline Social function & 2 & 53.76 & 23.85 & 2.60 & 31.60 & 0.849 \\
\hline Energy/fatigue & 4 & 42.76 & 22.22 & 0.00 & 7.90 & 0.837 \\
\hline Overall Score & & 51.01 & 18.38 & 0.00 & 0.00 & - \\
\hline
\end{tabular}

Table 4: Central tendency, variability (including floor and ceiling effects), reliability of KDQOL-SFTM scales at end of treatment visit.

\begin{tabular}{|lllllll|}
\hline Kidney disease-targeted scale & Mean & $\begin{array}{l}\text { Std } \\
\text { deviation }\end{array}$ & $\%$ Floor & \% Ceiling & $\begin{array}{l}\text { Internal consistency } \\
\text { reliability }\end{array}$ & p-value \\
\hline Burden of kidney disease & 29.53 & 21.55 & 7.89 & 0.00 & 0.651 & 0.28 \\
\hline Cognitive function & 62.24 & 23.16 & 2.63 & 2.60 & 0.665 & 0.99 \\
\hline Effect of kidney disease & 69.79 & 22.67 & 0.00 & 10.50 & 0.690 & 0.89 \\
\hline Sleep & 70.97 & 19.30 & 0.00 & 0.00 & 0.660 & 0.07 \\
\hline Quality of social interaction & 63.80 & 22.39 & 2.63 & 5.30 & 0.650 & 0.35 \\
\hline Symptom/problem list & 61.82 & 18.95 & 0.00 & 2.60 & 0.669 & 0.41 \\
\hline Social support & 63.13 & 31.54 & 2.63 & 31.60 & 0.640 & 0.58 \\
\hline Work status & 31.58 & 35.68 & 50.00 & 10.50 & 0.701 & 0.16 \\
\hline 36 item health survey & & & & & & 0.229 \\
\hline Physical function & 49.73 & 25.66 & 2.60 & 2.60 & 0.837 & 0.204 \\
\hline Role physical & 34.24 & 35.11 & 34.20 & 15.80 & 0.782 & 0.08 \\
\hline Pain & 53.82 & 29.17 & 5.30 & 13.20 & 0.778 & 0.009 \\
\hline General Health & 41.21 & 19.57 & 2.60 & 0.00 & 0.791 & 0.914 \\
\hline Emotional wellbeing & 57.34 & 20.35 & 2.60 & 0.00 & 0.803 & 0.47 \\
\hline Role emotional & 40.30 & 45.32 & 50.00 & 31.60 & 0.765 & 0.09 \\
\hline Social function & 5.18 & 19.36 & 0.00 & 0.00 & 0.825 & 0.581 \\
\hline Energy/Fatigue & 47.50 & 21.87 & 0.00 & 0.00 & 0.777 & \\
\hline Overall score & 52.76 & 17.05 & 0.00 & 0.00 & - & \\
\hline
\end{tabular}




\section{Quality of life assessment}

KDQOL-SFTM1.3 (SF-36 modified for CKD and Dialysis) composed of 43 kidney-specific items and 36 general health items was used for assessing quality of life. Comparison of KDQOL-SFTM means score values before and after treatment revealed significant improvement in general health, $\mathrm{p}<0.05$. Sleep, pain, energy/fatigue status also showed impressive improvement though not significant, Table 3 and 4. However overall health score was almost same which may be attributed to short duration of therapy. 36-item health survey scales had a Cronbach's $\alpha$ above the recommended minimum value of 0.7 to indicate good reliability. SGA scores showed an average improvement from baseline by 2 points.

\section{Safety parameters}

The orally administered study product was found to be safe and well tolerated as none of the study patients complained of serious adverse reaction. Two patients complained of bloating but continued the treatment and completed the study. None of the patients withdrew from the study as a result of adverse drug reaction.

\section{DISCUSSION}

The gut microflora has co-evolved with humans over thousands of years for a mutually beneficial coexistence and plays an important role in individuals health and disease. ${ }^{19}$ The human gut harbors 100 trillion microbial cells, which influence the nutrition, metabolism, physiology, and immune function of the host. ${ }^{20}$ Maintaining this ecosystem (Gut milieu) in a balanced state is an important requisite for maintenance of health.

In CKD with dysbiosis, maintaining the gut milieu may translate to down regulation of uremic toxin production and their absorption in small and large intestines. Uremic dysbiosis causes metabolic disruption, with up-regulation of inflammatory state and increased absorption of uremic toxins. This in turn translates to progression of CKD and worsening of metabolic syndrome sequel that eventually promotes high morbidity and mortality among CKD patients.

The research initiatives on role of probiotics in CKD have increased over the last decade with the goal of exploring their therapeutic potentials. There is growing evidence towards efficacy of this therapy in, retarding progression and reducing complications of CKD. Fundamental principles of dietetic practice is to recommend natural food sources as first line therapy, and it is only when this fails that supplements are used. ${ }^{10}$ End Stage Renal Disease patients have very poor appetite and intake; they are very unlikely to get the load of these specific strains of probiotics from natural food and hence require supplementation.
Moreover the probiotics are highly strain and dose specific. Increasing dose of Streptococcus thermophilus would improve creatinine without improvement in general condition and increasing dose of bifidobacteria would improve inflammatory state and probably reduce mortality.

One of the major factors contributing to increased morbidity and mortality among dialysis patients is systemic inflammation which increases risk of CVD associated mortality. hsCRP, TNF- $\alpha$ and IL- 6 are the key cytokines that mediate both acute and chronic inflammation. The levels of these cytokines progressively increases with stages of CKD being recorded highest in patients on dialysis. ${ }^{21}$ Framingham Offspring cohort was recorded to have significantly elevated level of TNF- $\alpha$ and IL- 6 among patients with CKD. ${ }^{22}$

In our study, use of symbiotic among dialysis patients showed a reduction in hsCRP and TNF- $\alpha$ levels from baseline in $60.52 \%, 55.26 \%$ patients respectively. IL-6 levels were reduced in $44.7 \%$ of treated patients though the degree of decline was not significant. Supplementation of symbiotic in stage 5D CKD may be a step towards achieving a near normal gut biota which in turn normalizes the gut milieu in CKD patients. This translates to reduction in inflammatory markers and improvement in other metabolic parameters as shown in our study.

Natarajan et al in a double-blind, placebo-controlled trial with 21 peritoneal dialysis patients reported probiotics taken daily for 6 months was effective in reducing serum levels of TNF- $\alpha$ and IL-6, both pro-inflammatory cytokines. They observed a tendency for a decrease in Creactive protein in 22 MHD patients after 8 weeks of probiotics supplementation. $^{23}$

In our study $34.21 \%$ and $28.94 \%$ of patients had a significant decrease in BUN and urea levels from baseline. Decrease in creatinine levels of $28.9 \%$ of patient was not significant probably as they were in End Stage Renal Disease (ESRD). Our study showed that among responders there was a statistically significant improvement in parameters of metabolic importance likeUric acid, total cholesterol, LDL, TGL, VLDL, HDL, hemoglobin, hematocrit. An improvement in these parameters of metabolic importance may translate to reduce morbidity \& mortality among patients with ESRD.

Our study probably paves the path for a double blind RCT involving large number of study subjects to look at role of symbiotic for improving long term survival and improved cardiovascular morbidity and mortality. Similar probiotic supplementation study by Natarajan et al in CKD patients had shown statistically significant reduction in BUN $(\mathrm{P}<0.05)$, uric acid level $(\mathrm{p}>0.05)$ and creatinine ( $p>0.05$ ) levels of $63 \%, 33 \%$ and $43 \%$ of predialysis study subjects. ${ }^{24}$ 
Natarajan et al showed a statistically significant improvement in hemoglobin, hematocrit, creatinine and C-reactive protein, post treatment in a "dose escalation, safety and impact of a strain specific probiotic study in CKD patients. ${ }^{1}$

Health related quality of life (HRQOL) is used almost exclusively in clinical studies, with the nephrology community increasingly realizing the potential importance of HRQOL assessment in the clinical care of its patients. The scores provide additional information on the individual's wellbeing beyond the information gained from the patient's clinical and laboratory assessments. The Kidney Disease Quality of Life Questionnaire-Short Form is the most widely used QOL measures for CRF patients. Measuring the impact of a treatment on patients' quality of life is an important outcome measure. The main aim of treatment in patients with CKD Stage 5D is to improve QOL, physical activity and emotional wellbeing with reduction in Protein Energy Wasting $(\mathrm{PEW}){ }^{18}$

Comparison of KDQOL-SF ${ }^{\mathrm{TM}}$ mean score values before and after treatment in this study revealed a significant improvement in general health, closely followed by sleep, pain, energy/fatigue, but the overall health score was almost same. This may be attributed to the fact that the course of treatment was only 6 weeks. Significant improvement in quality of life indicators would probably require longer duration of treatment. One patient had healthy weight gain of $12 \mathrm{~kg}$ in 4 wks in our study.

Other published studies had shown a similar trend, Natarajan et al in their study with probiotic dietary supplementation in CKD showed $85 \%$ subjects expressed a substantial higher perceived QOL during the treatment period, the study used a customized QOL questionnaire on a subjective scale of 1-10 rather than the generally used SF36 form. ${ }^{24}$ Ranganathan et al in their study used SF36 and showed significant improvement in physical functioning, a trend toward reduction of pain, with no significant change in mental, emotional and social wellbeing. ${ }^{1}$

\section{CONCLUSION}

This study establishes the safety and anti-Inflammatory efficacy of this symbiotic (probiotic and prebiotic) supplementation in patients with Stage 5D CKD on twice a week dialysis. To our knowledge this is the first study looking at anti-inflammatory role of symbiotic in CKD Stage 5D patients. The study suggests that a randomized, placebo controlled, double blinded study with a large sample size is warranted in future to establish the efficacy of symbiotic in CKD patients.

\section{ACKNOWLEDGEMENTS}

Author would like to thank all patients who took part in the study, the study staff, nurse and physicians for their assistance in collecting data. Authors are grateful to professor Dr. Shamshad Begum, JJM Medical College, Davangere, India for carrying out the statistical analysis for this study. This work was supported financially by Mylin Biotech India Private limited, Bangalore, India. The trial was designed, conducted, analyzed, and interpreted by the investigators independent of funding sources. The tests were done by reputed and reliable $3^{\text {rd }}$ party, SRL lab, which was independent of the data collection center, investigators and Mylin Biotech.

Funding: Mylin Biotech India Private limited, Bangalore, India

Conflict of interest: None declared

Ethical approval: The study was approved by the Institutional Ethics Committee

\section{REFERENCES}

1. Ranganathan N, Pechenyak B, Vyas U. Dose Escalation, Safety and Impact of a Strain-Specific Probiotic (Renadyl ${ }^{\mathrm{TM}}$ ) on Stages III and IV Chronic Kidney Disease Patients. J Nephrol Ther. 2013;3:2-9.

2. Sicree R, Shaw J, Zimmet P. Diabetes and impaired glucose tolerance. In: Gan D, Diabetes Atlas. 3rd ed. Brussels, International Diabetes Federation. 2006;15109.

3. Modi GK, Jha V. The incidence of end stage renal disease in India. A population based study. Kidney Int. 2006;70:2131-33.

4. Singh AK, Farag YM, Mittal BV. Epidemiology and risk factors of chronic kidney disease in India results from the SEEK (Screening and Early Evaluation of Kidney Disease) study. BMC Nephrol. 2013;14:114.

5. Ballal SH. The burden of chronic kidney disease in a developing country, India by quest. Davita's clinical journal for nephrologists. 2007;9:12-9.

6. Koppe L, Mafra D, Fouque D. Probiotics and chronic kidney disease. Kidney Int. 2015;88:958-66.

7. Ramezani A, Dominic SR. The Gut Microbiome, Kidney Disease, and Targeted Interventions. J Am Soc Nephrol. 2014;25:657-70.

8. Vaziri ND, Wong J, Pahl M. Chronic kidney disease alters intestinal microbial flora. Kidney Int. 2013;83:308-15.

9. Hida M, Aiba Y, Sawamura S. Inhibition of the accumulation of uremic toxins in the blood and their precursors in the feces after oral administration of Lebenin, a lactic acid bacteria preparation, to uremic patients undergoing hemodialysis. Nephron. 1996;74:349-55.

10. Rossi M, Johnson DW, Campbell KL. The KidneyGut Axis: Implications for Nutrition Care. Journal of Renal Nutrition. 2015:1-5.

11. Vitetta L, Linnane AW, Gobe GC. From the Gastrointestinal Tract (GIT) to the Kidneys: Live Bacterial Cultures (Probiotics) Mediating Reductions 
of Uremic Toxin Levels via Free Radical Signaling. Toxins. 2013;5:2042-57.

12. Evenepoel P, Meijers BK, Bammens B. Uremic toxins originating from colonic microbial metabolism. Kidney Int. 2009;114:S12-9.

13. Kotanko P, Carter M, Levin NW. Intestinal bacterial microflora-a potential source of chronic inflammation in patients with chronic kidney disease. Nephrol. Dial. Transplant. 2006;21:2057-60.

14. Schepers E, Glorieux G, Vanholder R. The gut: the forgotten organ in uremia. Blood Purif. 2010;29:1306.

15. Vitetta L, Gobe G. Uremia and chronic kidney disease: The role of the gut microflora and therapies with pro- and prebiotics. Mol. Nutr. Food Res. 2013;57:824-32.

16. Gibson GR, Roberfroid MB. Dietary modulation of the human colonic microbiota: introducing the concept of prebiotics. J Nutr. 1995;125:1401-12.

17. Vitetta L, Sali A. Probiotics, prebiotics and gastrointestinal health. Med. Today. 2008;9:65-70.

18. Kuriokose AV, Kenchaappa V, Kumar S. Assessment of quality of life in chronic renal failure patients in India, ISSN 2029-9575, Quality Issues and Insight in the $21^{\text {st }}$ century; 1,2 .

19. Hooper LV, Gordon JI. Commensal host bacterial relationships in the gut. Science. 2001;292:1115-18.
20. Bäckhed F, Ley RE, Sonnenburg JL. Host-bacterial mutualism in the human intestine. Science. 2005;307:1915-20.

21. Lee1 BT, Ahmed FA, Hamm LL. Association of Creactive protein, tumor necrosis factor-alpha, and interleukin-6 with chronic kidney disease. BMC Nephrology. 2015;16:77.

22. Upadhyay A, Larson MG, Guo CY. Inflammation, kidney function and albuminuria in the Framingham Offspring cohort. Nephrol Dial Transplant. 2011;26:920-26.

23. Natarajan R, Pechenyak B, Vyas U. Randomized controlled trial of strain-specific probiotic formulation (Renadyl) in dialysis patients. BioMed Res Int. 2014;568-71.

24. Natarajan R, Ranganatha P, Eli A. Pilot Study of Probiotic Dietary Supplementation for Promoting Healthy Kidney Function in Patients with Chronic Kidney Disease. Adv Ther. 2010;27(9):634-47.

Cite this article as: Srinivasa S, Madhusudhan SK. A prospective study to evaluate the safety and efficacy of symbiotic supplementation (probiotic and prebiotic combination) in stage 5D chronic kidney disease patients. Int J Basic Clin Pharmacol 2017;6:765-73 